
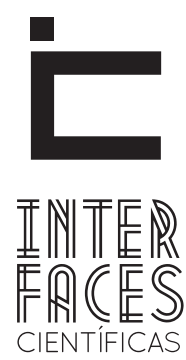

DIREITO

\title{
CAPACIDADE FUNCIONAL PARA O TRABALHO: IMPORTANTE INSTRUMENTO DE DECISÃO PARA A JUSTIÇA DO TRABALHO
}

\author{
Ronaldo Veronesi ${ }^{1}$
}

\section{RESUMO}

A capacidade funcional para o trabalho é um importante instrumento para a decisão judicial dentro dos processos trabalhistas. Nos dias atuais os peritos judiciais utilizam métodos convencionais, baseado em suas experiências profissionais e conhecimento sobre doenças, de forma observacional para quantificar a capacidade funcional do reclamante. Esta pesquisa desenvolveu e validou um protocolo de avaliação da capacidade funcional para perícia judicial do trabalho. Foram escolhidos 10 casos periciais, para serem avaliados por 44 profissionais, fisioterapeutas, pós graduandos em Fisioterapia do Trabalho, que tiveram treinamento em perícia judicial e que fizeram pericias em sua carreira. Dois grupos foram escolhidos aleatoriamente, onde um foi treinado e capacitado para utilizar o protocolo desenvolvido nesta pesquisa, chamado de estudo e o outro grupo (controle) utilizou o método tradicional, de forma observacional. O grupo controle apresentou uma grande variabilidade nos resultados (CVQ $=33 \%)$, enquanto o estudo apresentou baixa variabilidade (CVQ=6\%). Os grupos apresentaram diferenças estatísticas entre seus resultados $(\mathrm{p}<0,0001)$. O teste Kendall W apresentou alta concordância entre os avaliadores do estudo, 91,7\%, concluindo que é confiável. Já Kendall Tau, mostrou que tem repetibilidade quase perfeita. A presente pesquisa mostrou que o protocolo é mais fiável e com menor variabilidade entre seus resultados, auxiliando a justiça ser mais justa.

\section{PALAVRAS CHAVES:}

Incapacidade Funcional. Capacidade laboral. Perícias. 


\section{ABSTRACT}

The functional capacity to work is an important tool for the ruling within the labor process. Nowadays judicial experts using conventional methods, based on their professional experiences and knowledge on diseases, observational way to quantify the functional capacity of the claimant. This research developed and validated a protocol for assessing functional capacity for judicial investigation work. Ten forensic cases were selected for further evaluation by 44 professionals, physiotherapists, graduate students in Physiotherapy Labour, who had training in legal skills and expertise in that made his career. Two groups were randomly chosen, where one is trained and qualified to use the protocol developed in this research, called the study and the other group (control) used the traditional method, observational manner. The control group showed a great variability in results (CVQ $=33 \%)$, while the study showed low variability (CVQ $=6 \%)$. Groups showed statistical differences between their results $(p<0.0001)$. The Kendall $W$ test showed high agreement among raters in the study, $91.7 \%$, concluding that it is reliable. Kendall Tau showed he has almost perfect repeatability. This research demonstrated that the protocol is more reliable and less variability among their results, helping justice to be fairer.

\section{KEYWORDS:}

Functional Disability. Work Capacity. Skills.

\section{RESUMEN}

La capacidad funcional para el trabajo es una herramienta importante para la resolución en el proceso de trabajo. Hoy en día, los expertos judiciales utilizan métodos convencionales, basados en sus experiencias profesionales y conocimientos sobre las enfermedades, de manera observacional para cuantificar la capacidad funcional de la demandante. Esta investigación ha desarrollado y validado un protocolo para evaluar la capacidad funcional para el trabajo de investigación judicial. Fueron seleccionados 10 casos forenses para su posterior evaluación por 44 profesionales, fisioterapeutas, estudiantes de postgrado en Fisioterapia del Trabajo, que tenían formación en conocimientos jurídicos y experiencia en que hizo su carrera. Dos grupos fueron escogidos al azar, el grupo estudio, recibió un entrenamiento para utilizar el protocolo desarrollado en esta investigación, y el otro grupo (control) se utiliza el método tradicional, de manera observacional. El grupo control mostró una gran variabilidad en los resultados (CVQ $=33 \%$ ), mientras que el estudio mostró una baja variabilidad (CVQ $=6 \%$ ). Los grupos mostraron diferencias estadísticas entre los resultados ( $p<0,0001)$. La prueba de Kendall W mostró una alta concordancia entre los evaluadores en el estudio, el 91,7\%, llegando a la conclusión de que es confiable. La prueba de Kendall Tau, demostró que la repetición del protocolo es casi perfecta. Esta investigación demostró que el protocolo es más fiable y menos variabilidad entre sus resultados, ayudando a la justicia para ser más justa.

\section{PALABRAS CLAVES}

Discapacidad funcional. Capacidad de trabajo. Pericias. 


\section{INTRODUÇÃO:}

A palavra trabalho vem do latim, tripalium, que significa um instrumento de tortura usado pelos romanos para obrigar os escravos a trabalhar. 0 sofrimento foi dando lugar ao esforçar, lutar até chegar à palavra trabalhar.

Com o Capitalismo instituído no mundo global, o trabalho passou a ser dado como um fator de aquisições, de recursos, bens materiais e de riqueza. Com o sistema capitalista o dinheiro passou a ser o principal fruto de conquista do homem, e seus valores são dados para os bens materiais. Os princípios filosóficos e imateriais do trabalho, o benefício psicoemocional que o trabalho honesto e prazeroso traz para o homem, bem como os valores da vida humana, com o mundo capitalista fica em segundo plano (VERONESI, 2013).

O trabalho é necessário para manter as condições da vida humana, é um fator importante para manter o bem estar psicossocial dos trabalhadores, que podem estar sujeitos a riscos e perigos das condições de trabalho e o ambiente de trabalho é um fator importante para manter ou destruir a saúde do trabalhador (VERONESI, 2009).

A dignidade humana constitui uma só realidade, indivisível, composta de partes física, moral, intelectual e emocional, interdependentes, que lhe conferem uma identidade única e irrenunciável. A pessoa humana é o bem mais valioso que a sociedade possui, um bem que não se pode traduzir facilmente ou integralmente em valor monetário (VERONESI, 2013).

Quando existe lesão ou dano ao indivíduo e este é o objeto de fundamentação da reclamação de indenização como consequência do acidente ou doença do trabalho, é necessário uma investigação técnica chamada de perícia judicial.

A perícia judicial é uma análise técnico-científica realizada por um especialista no assunto, com fim de aclarar aspectos técnicos fundamentais para a decisão do juiz (BERNARDES, 2011). Nos casos de acidentes ou doenças do trabalho, a perícia irá estabelecer o nexo causal bem como avaliar e quantificar a capacidade funcional residual para o trabatho (VERONESI 2013).

Segundo os estudos de Wind (2009) um protocolo de avaliação da capacidade funcional é uma importante ferramenta para avaliar a capacidade física para o trabalho, para avaliar o trabalhador antes de este retornar ao trabalho ou mesmo para avaliar as incapacidades em caso de reclamatória judicial.

A Organização Mundial de Saúde (OMS) tem proposto dois grandes modelos conceituais de incapacidade. 0 modelo médico que considera a incapacidade como um estado da pessoa, causado diretamente por doença, trauma ou por qualquer outra condição de saúde. 0 outro é o modelo social de incapacidade, que por outro lado, considera a incapacidade como um problema de natureza social e de forma alguma como um atributo do indivíduo. Segundo o modelo social, a incapacidade exige uma resposta política, visto o problema decorrer da existência de um meio ambiente desajustado, formas de socioeconômica desequilibrada e criado por atitudes e outras condições do meio social interferindo de forma direta e ou indireta na incapacidade funcional (CIF, 2003).

O conceito de capacidade de trabalho em relação com a capacidade do trabalhador para realizar seu trabalho de acordo com as exigências da tarefa, sua saúde e suas capacidades físicas e mentais, pode ser considerada como o resultado de um processo dinâmico entre os recursos individuais em relação com seu trabalho, se vê relacionada com vários fatores, tais como as características sociodemográficas, estilo de vida, processo de envelhecimento e as exigências do trabalho (MARTINEZ, 2006). Sampaio (1999) 
em seu estudo analisou os seguintes dados dentro da avaliação da capacidade funcional para o trabalho: dados sociodemográfico (idade, sexo, estado civil, escolaridade).

Devido a grande responsabilidade dada à conclusão do laudo pericial, para a decisão judicial, se torna fundamental que se tenha um instrumento cientificamente comprovado para dar uma segurança e eficiência nos laudos periciais.

\section{MÉTODOS}

A presente pesquisa é fruto da tese de doutorado do autor, sendo aprovada em banca presencial, pelo Instituto Universitário Italiano de Rosário. A pesquisa foi aprovado pelo comitê de ética local em 16 de abril de 2012. Foi desenvolvida por meio de um estudo analítico experimental controlado e aleatorizado. Foi escolhido dez periciais aleatoriamente, que foram avaliadas por dois grupos, cada um com vinte e dois avaliadores. 0 grupo controle avaliou a capacidade funcional dos periciados de maneira convencional, já o grupo estudo utilizou o protocolo desenvolvido nesta pesquisa. 0 critério de inclusão foi que os avaliadores eram fisioterapeutas, pós-graduandos em Fisioterapia do Trabalho, que fizeram uma capacitação em perícia judicial e que já tinham feito uma perícia judicial do trabalho. Todos os 44 avaliadores desta pesquisa foram listados numericamente de 1 a 44 e depois sorteados pelo software Epidat ${ }^{\circledR}$, sendo separados assim os dois grupos de estudo.

O grupo de estudo utilizou o protocolo criado nesta pesquisa para avaliar a capacidade funcional para o trabalho dos 10 casos periciais, já o controle, os sujeitos avaliaram a capacidade funcional dos mesmos 10 casos periciais de maneira convencional, de forma observacional e subjetiva.

Esta investigação foi dada em duas etapas, a primeira foi a construção do protocolo de avaliação da capacidade funcional para perícia judicial do trabalho e a segunda foi a validação deste protocolo. Esta validação foi feita por meio de uma análise estatística comparativa entre os grupos estudados.

O protocolo foi construído a partir de aspectos fundamentais para avaliar a capacidade funcional do trabalho dentro de uma filosofia de pensamento referente à funcionalidade do trabalhador, baseado em dois instrumentos: Classificação Internacional de Funcionalidade (CIF) e a classificação de Funcionalidade de Baremo. Além destes dois instrumentos foram utilizados dados para a análise da capacidade funcional como: idade, escolaridade, estruturas lesionadas, prognóstico das doenças e testes funcionais.

Foi feito um protocolo para análise dos membros superiores e outro para os membros inferiores e coluna vertebral, devido as variáveis da CIF ser específica para cada segmento. O protocolo foi criado em um sistema informatizado para melhor aplicabilidade e gestão dos dados. Possui 3 listas de análises: lista documental, prática (estas duas listas são comuns aos dois protocolos), e atividade e participação, que é específica para cada protocolo, onde tem elementos específicos de funcionalidade para cada segmento de análise relacionados e orientados pela CIF.

A lista documental é chamada assim, devido ter variáveis fixas, e que são comprovadas por documentos. As seguintes variáveis foram analisadas:

Variável idade: para esta variável foi estabelecido como parâmetro, a idade inicial permitida de trabaIho no Brasil, 18 anos, e a final de aposentadoria pela previdência social, 65 anos, onde quanto mais velho maior a incapacidade, condição esta comprovada cientificamente e de conhecimento notório.

Variável educação: nos dias atuais a educação é um fator fundamental para as oportunidades de trabalho, se estiver associado ainda com treinamento funcional específico, o campo de trabalho aumenta 
mais ainda. Para a teoria do Capital Humano, quanto maior for o grau de escolaridade, maiores serão as oportunidades no mercado de trabalho, isto dentro de um meio econômico equilibrado, havendo uma relação de causalidade (educação explicando as oportunidades). Sampaio (1999), em seu estudo utilizou para avaliar a capacidade funcional para o retorno ao trabalho, dentre outros os dados, a escolaridade que foi dividida em 8 categorias, as mesmas estabelecidas pela CIF e utilizada nesta pesquisa: analfabeto, semianalfabeto, primeiro grau completo, primeiro grau incompleto, segundo grau completo, segundo grau incompleto, curso universitário completo e curso universitário incompleto.

Variável Classificação da funcionalidade Baremo: é um instrumento utilizado pelo governo espanhol para classificar a capacidade funcional. Esta classificação é dividida em 5 categorias de acordo com sua incapacidade da seguinte forma: transtornos funcionais leves, moderados, médios, importantes e muito importante.

Na Variável Estrutura lesionada: foi utilizado como uma classificação gradual de 1 a 10, quanto maior o número de estruturas afetadas maior será a pontuação atribuída e consequentemente maior a incapacidade, interpretação utilizada tanto pela SUSEP, quanto Baremo (CRIADO DEL RÍO, 1999).

A última variável desta lista é analise Prognóstica da lesão: de acordo com os Parâmetros de dano corporal perante as valorizações indenizatória segundo $\mathrm{Ma}$ galhães (2003) e Vieira (2001), pode-se ter as seguintes condições: Incapacidade temporária e Permanente. 0 estudo feito por Baéssa (2013) com fisioterapeutas de vários estados do Brasil mostra que, de acordo com a característica patogênica da doença, quando temporária, esta tem previsão de recuperação, e estas previsões estão presentes nesta variável analisada.

A lista prática do protocolo, consta de 3 provas funcionais orientadas pela $\mathrm{CIF}$, onde é analisado o comportamento funcional do segmento que sofreu o dano de maneira dinâmica, estática e a angulação máxima de movimento obtida (VERONESI, 2012). Os participantes do grupo estudo, que utilizou o protocolo desta pesquisa, recebeu um treinamento específico para análise do comportamento funcional do movimento humano para analise e interpretação destas provas funcionais.

Para provas que analisam o comportamento funcional do segmento de forma dinâmica e estática, os sujeitos foram treinados para analisar o processo de simulação ativa, situação esta encontrada quando o reclamante tenta simular na perícia para induzir o perito. Quando identificada a presença da simulação ativa no processo pericial, o reclamante recebia a menor pontuação na tabela, devido a falta de elementos idôneos de avaliação. Para fazer a análise da angulação máxima do movimento, os sujeitos do grupo estudo foram treinados a utilizar um programa de computador para a medição, enquanto o grupo controle, que avaliou os casos de forma subjetiva, avaliaram a movimentação de forma observacional.

A terceira lista do protocolo é uma categoria da CIF com o código D4100-6405, que trata das atividades e participações do avaliado. Em ambos os protocolos a lista de atividade e participação possui 29 itens referente a condições funcionais do dia a dia direcionados para cada segmento analisado.

\section{RESULTADOS}

0 teste de Wilcoxon mostrou que existe significância estatística $(p<0,0001)$ entre os dois grupos. 0 teste bilaterial $(p<5 \%)$ mostrou que existe diferença significativa entre os dois grupos (Tabela 1). Isso mostra que o novo método produz resultados diferentes do método existente. 0 teste Kendall $W$ apresentou alta concordância entre os avaliadores do estudo, 91,7\%, concluindo que é confiável. Já Kendall Tau, mostrou que tem repetibilidade quase perfeita. 
Tabela 1- Resultados do teste de Wilcoxon, quando comparado os grupos estudados

\begin{tabular}{l}
\hline STDUY GROUP - CONTROL GROUP \\
\hline$-3,459 a$ \\
, 000
\end{tabular}

a. Based on negative ranks.

b. Wilcoxon Signed Ranks Test

O grupo controle possuiu um coeficiente de variação interquatil CVQ igual a $33 \%$ enquanto o grupo estudo apresentou somente $6 \%$.

Tabela 2- D istribuição do coeficiente de variação interquatil dos grupos estudados

\begin{tabular}{lll} 
& Grupo Controle & Grupo Estudo \\
\hline Mediana & $30 \%$ Incapacidade & $20 \%$ Incapacidade \\
Mínimo & $100 \%$ Capacidade & $10 \%$ Incapacidade \\
Máximo & $100 \%$ Incapacidade & $60 \%$ Incapacidade \\
CVQ & $33 \%$ & $6 \%$ \\
N & 220 & 220 \\
\hline
\end{tabular}

O controle apresentou grande variabilidade enquanto o grupo estudo possuiu baixa variabilidade.

Figura 1- Variabilidade entre os casos estudados entre os grupo
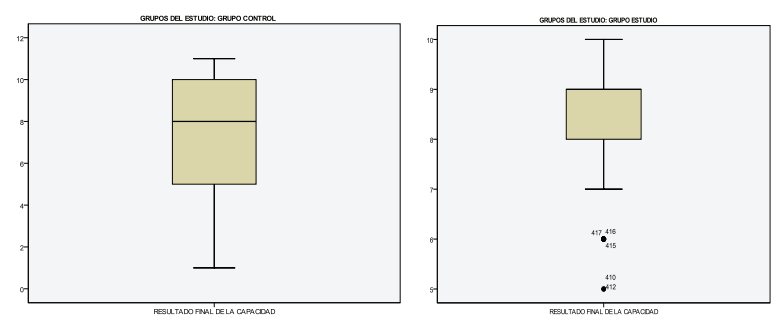

A baixa variabilidade no grupo estudo também é observada quando comparamos os grupos em cada caso. 0 grupo controle possuiu CVQ maior que $30 \%$ com excessão dos casos 2, 6 e 9 (Tabela 3).

Tabela 3 - Análise do Coeficiente de Variação Interquatil de todos os casos no grupo controle

\begin{tabular}{lllll}
\hline \multicolumn{5}{c}{ GRUPO CONTROLE } \\
\hline Casos & $1^{\circ} \mathrm{Q}$ & Mediana & $30 \mathrm{Q}$ & CVQ \\
CASO 1 & $20 \%$ & $30 \%$ & $40 \%$ & $33 \%$ \\
CASO 2 & $60 \%$ & $70 \%$ & $78 \%$ & $13 \%$ \\
CASO 3 & $0 \%$ & $15 \%$ & $28 \%$ & $100 \%$ \\
CASO 4 & $40 \%$ & $70 \%$ & $80 \%$ & $33 \%$ \\
CASO 5 & $0 \%$ & $0 \%$ & $20 \%$ & $100 \%$ \\
CASO 6 & $50 \%$ & $55 \%$ & $68 \%$ & $15 \%$ \\
CASO 7 & $0 \%$ & $25 \%$ & $50 \%$ & $100 \%$ \\
CASO 8 & $0 \%$ & $20 \%$ & $30 \%$ & $100 \%$ \\
CASO 9 & $45 \%$ & $80 \%$ & $80 \%$ & $28 \%$ \\
CASO 10 & $3 \%$ & $20 \%$ & $20 \%$ & $78 \%$ \\
\hline
\end{tabular}

Para melhor ilustrar a variabilidade dos resultados da capacidade funcional entre os avaliadores dos dois grupos, foi utilizado gráficos de linhas, onde demonstra a distribuição uniforme do grupo estudo nos casos analisados enquanto o grupo estudo apresentou uma distribuição variável.

Figura 2 - Gráfico de linha mostrando a variabilidade dos resultados nos grupos estudados nos casos 1 e 2 .

Caso 1

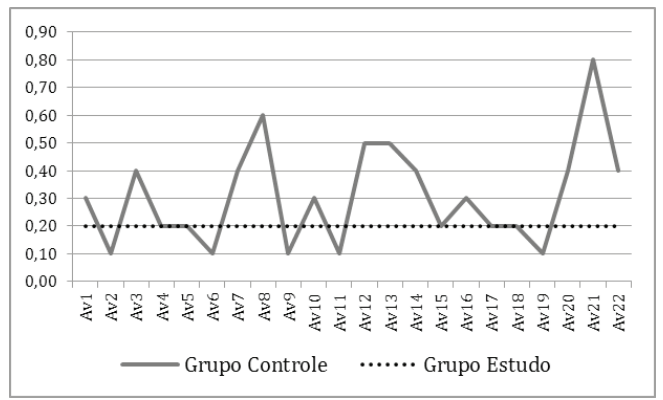


Caso 2

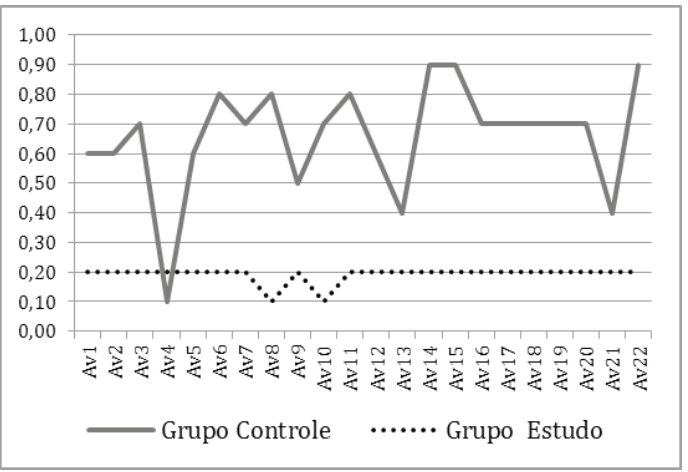

\section{DISCUSSÃO}

Fernandes (2005), em seu trabalho já mostrava a importância do resultado da capacidade funcional para a conclusão judicial, por estar referido a quantias financeiras.

O grupo controle, que avaliou a capacidade funcional de maneira convencional, de forma observacional e subjetiva, apresentou uma grande variabilidade nos resultados dos mesmos casos periciais feito por distintos profissionais (CVQ 33\%). Enquanto o grupo estudo praticamente não apresentou (CVQ 6\%), mostrando que o protocolo desenvolvido nesta pesquisa é mais sólido e consistente em seus resultados.

Recai sobre o empregador o dever de adotar medidas para eliminar os riscos de acidentes (art. 155 da CLT). Quando o empregador viola seu dever e fere o direito do empregado, causando uma doença do trabalho, fica obrigado ao empregador a reparar o dano causado.

Havendo redução da capacidade laborativa, a reparação de dano material corresponderá às despesas de tratamento e a uma pensão correspondente à importância da depreciação sofrida pelo trabalhador segundo o art. 950 do Código Civil Brasileiro de 2002.
No contexto da justiça do trabalho, nas ações em que visam a reparação de danos decorrentes a doenças laborais ou acidentes de trabalho, os lucros cessantes são "parcelas mensais de indenização decorrente a perda da capacidade funcional" (OLIVEIRA, 2006).

Nesta reflexão onde o valor da incapacidade funcional gera o valor da indenização, e que nesta pesquisa mostrou que a avaliação da capacidade funcional, feita baseada nas experiências profissionais e dos conhecimentos técnicos sobre as doenças e perícia judicial do perito, que é a forma convencional atualmente, gera uma variabilidade do resultado entre $33 \%$. O que indicaria que, o valor indenizatório pode estar sendo injusto em $33 \%$ no seu montante final.

Isto fere os princípios da justiça, o termo justiça vem do latim iustita que de maneira simples quer dizer a igualdade entre todas as pessoas, a equidade, baseada em uma forma imparcial, balanceada e ponderada, vem do principio básico de preservar os direitos da sociedade, em um sentido mais amplo, pode ser considerado como um termo abstrato que designa o respeito ao terceiro e ao outro, a aplicação ou reposição do direito da virtude moral ou material.

O Estudo de Wind (2009) demonstrou que havia uma diferença significativa na conclusão sobre a capacidade funcional de avaliados feito por peritos holandeses, onde o grupo experimental, que foi capacitado em informações pertinente sobre a capacidade funcional, apresentou resultados mais satisfatórios e igualitários quando comparado com o grupo controle, que não tinha recebido capacitação. Mostrando a importância da informação sobre a capacidade funcional para sua avaliação e a necessidade de fazer uma avaliação baseada em um instrumento científico.

Uma das justificativas para que o grupo estudo tenha resultados com baixa variabilidade e o controle com variabilidade considerável, é que o grupo estudo recebeu uma capacitação específica sobre o processo 
de simulação ativa. Para Veronesi (2013), elementos de convencimento do perito são respostas de testes e observações feitas pelo perito para análise do processo de confiabilidade do comportamento funcional do movimento, o que indicaria uma possível condição de simulação feita pelo periciado.

Desta forma esta pesquisa vem confirmar os estudos de Wind (2009), que provou que a capacitação especifica do perito é fundamental para um melhor desempenho e um resultado mais fiável e justo.

\section{CONCLUSÃO}

Considerando que, os juízes decidem sobre os valores de indenização baseado na incapacidade funcional, torna claro e evidente que se ter um protocolo para avaliação da capacidade funcional para perícias judiciais confiáveis e seguro cientificamente, como foi provado nesta pesquisa, ter-se-á os princípios da justiça sendo aplicados e uma justiça mais justa!

Este protocolo, também, será muito útil na previdência social, auxiliando o processo pericial para análise da capacidade funcional para trabalho. Pode, também, ser muito útil pelas empresas junto aos programas de prevenção e controle da saúde ocupacional, como um instrumento de avaliação e controle da capacidade funcional do trabalhador.

Até o momento não existia um instrumento cientificamente comprovado para avaliar a capacidade funcional do periciado para a justiça do trabalho. Desta forma este protocolo vem iniciar um processo contínuo de melhorias, onde servirá de referência e ponto de partida para a construção de outros, até mesmo, para o aperfeiçoamento deste, aplicando assim o principio da lógica das ciências, fazer ciência para estimular a própria ciência.

\section{REFERÊNCIAS}

BAÉSSA, E.G.; VERONESI JR, J. R. Estudo sobre tempo e custos para reabilitação de lesões osteomusculares relacionados a justiça trabalhista. In: Congresso Brasileiro de Fisioterapia do Trabalho, 4, 2013, Juiz de Fora. Anais...Juiz de Fora, Universidade Federal de Juiz de fora, 2013.

BERNARDES, J.M., VERONESI, J. R. JR. A Atuação do Fisioterapeuta nas Perícias Judiciais de LER/DORT. Fisioterapia Brasil, v.12, n.3, maio/junho 2011.

CIF - Classificação Internacional de Funcionalidade, Incapacidade e Saúde, Centro Colaborador da OMS para a Classificação de Doenças em Português, S. Paulo, EdUP, 2003

CLT - Consolidação das Leis Trabalhistas do Ministério do Trabalho. Disponível em: <http://portal.mpt. gov.br/wps/wcm/connect/7281a48046792e1bbca1f f757a687f67/clc.pdf?MOD=AJPERES\&CACHEID=72 81a48046792e1bbca1ff757a687f67>. Acesso em: 2 mar. 2013.

CRIADO DEL RÍO, M.T. Valor ación médico-legal del daño de la persona. Civil, penal, laboral e administrativa: responsabilidad profesional del perito médico. Madrid: COLEX; 1999, cap.2, p.35-705.

FERNANDES, F. C.; CHEREM, A. J. Dano corporal e mensuração da incapacidade. Rev. Bras. Med. Trab., Belo Horizonte, v.3, n.2, p.123-34, ago-dez. 2005.

INSTITUTO NACIONAL DE SEGURIDADE SOCIAL INSS. A Previdência Social. Brasília, 2012. Disponível em: <http://www.mpas.gov.br>. Acesso em: 25 jan. 2012.

MAGALHÃES, T. Estudo tridimensional do dano em direito civil: lesão, função e situação (suaaplicaçãomédico-legal). Coimbra: Almedina; 1998. cap.1, p.24-83. 
MARTINEZ, M.C.; LATORRE M. R. D. O. Saúde e capacidade para o trabalho em trabalhadores de área administrativa. Rev. Saúde Pública, 2006; 40(5):851-8.

OLIVEIRA, S. G. Indenizações por Acidente de Trabalho e Doença Ocupacional. 2.ed. São Paulo: Ltr., 2006, p. 71.

SAMPAIO, R. F.; NAVARRO, A. G.; MARTÍN, M. M. Cad. Saúde Pública, Rio de Janeiro, 15(4):809-815, out-dez. 1999.

SQUILLA, L. S. Capacidade funcional dos portadores de hemiplegia por seqüela de acidente vascular cerebral: relação com assistência á saúde e participação da família. 2004. 133f. Dissertação (Mestrado em Saúde da Comunidade) Departamento de Medicina Social da Faculdade de Medicina de Ribeirão Preto da Universidade de São Paulo. Ribeirão Preto, SP.

VERONESI, J. R., Jr. Perícia Judicial para Fisioterapeutas: pericia técnica cinesiológica-funcional, assistência técnica judicial, modelos e legislações. São Paulo: Andreoli, 2009.
VERONESI, JR, J. R. La cuantificación de la capacidad funcional de los expertos Veronesi método de evaluación de experiencia judicial. Revista Terapia Manual, 2010, ago; 38:162-167.

VERONESI, JR, J. R. Testes Ortopédicos Funcionais para fisioterapeutas. São Paulo: Andreoli, 2012.

VERONESI JR, J. R. Perícia judicial para fisioterapeutas. 2.ed., São Paulo: Andreoli, 2013.

VIEIRA, D. N. P. A missão da avaliação do dano corporal em direito civil. Revista Sub Judice. Justiça e sociedade, maio 2001; v.7, p.23-31.

WIND, H; GOUTTEBARGE, V.; KUIJER, P. P. F. M.; SLUITER, J. K.; FRINGS-DRESEN, M. H. W. Effect of Functional Capacity Evaluation information on the judgment of physicians about physical work ability in the context of disability claims. Int Arch Occup Environ Health, 2009, 82:1087-1096. Dourados-MS, defendeu seu doutorado validando um protocolo de avaliação da capacidade funcional para perícia judicial, autor de 6 livros, 3 de perícia judicial, é professor do curso de aperfeiçoamento profissional em perícia judicial para fisioterapeutas e perícia ergonômica, é especialista em fisioterapia do trabalho e diretor da ABRAFIT. É coordenador dos cursos Aceito em: 12 de março de 2014 do IEDUV www.ieduv.com.br. veronesi@ieduv.com.br 